Nouvelles perspectives en sciences sociales

Revue internationale de systémique complexe et d'études relationnelles

\title{
Renouveler la science économique néo-classique? Prendre l'historicité au sérieux
}

\section{Jean Luc Demeulemeester et Claude Diebolt}

Volume 6, numéro 2, juin 2011

Sur le thème : « Les économistes dans la Cité »

URI : https://id.erudit.org/iderudit/1005773ar

DOI : https://doi.org/10.7202/1005773ar

Aller au sommaire du numéro

Éditeur(s)

Prise de parole

ISSN

1712-8307 (imprimé)

1918-7475 (numérique)

Découvrir la revue

Citer cet article

Demeulemeester, J. L. \& Diebolt, C. (2011). Renouveler la science économique néo-classique? Prendre l'historicité au sérieux. Nouvelles perspectives en sciences sociales, 6(2), 133-160. https://doi.org/10.7202/1005773ar
Résumé de l'article

Cet article est une critique interne de la discipline économique et une tentative de faire prendre conscience au sein de la communauté des économistes de l'importance de réintroduire l'histoire et l'historicité dans la discipline. 


\title{
Renouveler la science économique néo- classique? Prendre l'historicité au sérieux
}

\author{
Jean Luc Demeulemeester \\ Université libre de Bruxelles \\ Claude Diebolt \\ Université de Strasbourg, BETA/CNRS (UMR 7522)
}

Tes auteurs de ce texte sont deux économistes relativement Lorthodoxes - en ce sens qu'ils ont le souci de parler à leur communauté et sont bien conscients qu'adopter un discours trop en rupture n'aboutit qu'à rendre le message inaudible à ceux (les économistes) auxquels il est destiné. Cet article se voudra donc critique (au point que d'aucuns pourraient y voir une sempiternelle critique de l'économie a-temporelle qui sont de fait légions ${ }^{1}$ ), mais aussi constructif dans le cadre du paradigme dominant (interprété largement, mais restant dans la philosophie très " moderne » qui tend à considérer les phénomènes économiques comme des choses, dont l'analyse nécessite le passage par une modélisation mathématique, donc assez mécaniste). La critique semble essentielle à l'heure où la crise économique et financière semble mettre à mal les certitudes les mieux établies² (même si

$1 \quad$ Voir Graeme Donald Snooks, Economics without Time. A Science Blind to the Forces of Historical Change, Basingstoke, Macmillan, 1993; Graeme Donald Snooks (dir.), Historical Analysis in Economics, London, Routledge, 1993.

2 Voir à titre d'écho les actes d'un Symposium organisé en novembre 2009 dans le cadre des CESifo Economic Studies Conference au titre on ne peut plus évocateur: What's Wrong with Macroeconomics? (CESifo Economic Studies, vol. 
la majorité des économistes ne s'en rend pas toujours compte et certains voient dans la crise l'occasion de réaffirmer la pertinence des approches dominantes par exemple en macroéconomie avec les modèles d'équilibre général dynamiques stochastiques $\left.{ }^{3}\right)$. Mais cette critique se doit aussi d'être écoutée par la corporation des économistes. Si elle ne s'adresse qu'aux historiens, sociologues ou anthropologues, elle ne sera pas reçue par les économistes. Il nous semble pourtant qu'une place existe pour une science économique car l'analyse des systèmes et mécanismes économiques constitue en soi un domaine de recherche relativement autonome, et ni la sociologie économique ni l'anthropologie économique (malgré leurs très grands mérites par ailleurs) ne peuvent parfaitement s'y substituer.

Ce texte sera donc une critique interne de la discipline et une tentative de faire prendre conscience à la communauté des économistes de l'importance de réintroduire dans la discipline l'histoire et l'historicité (au sens bien spécifique que les économistes donnent à ce mot compte tenu de ce que, in fine, les concepts doivent en science économique pouvoir être opérationnalisés, c'est-à-dire représentés mathématiquement). Autant dire que la notion d'histoire que nous retiendrons ici sera très abstraite et mécaniste (une idée de dépendance à la trajectoire - nous nous inscrivons certainement dans la perspective néo-institutionnaliste qui irrigue aussi d'autres sciences sociales comme les sciences politiques ${ }^{4}$ ), même si en parallèle nous ne pourrons faire parfaitement l'économie du concept d'historicité tel que développé dans la philosophie ou les études littéraires ${ }^{5}$. En effet, c'est la

56, décembre 2010).

3 Voir Mickael Wickens, Macroeconomic Theory: A Dynamic General Equilibrium Approach, Princeton, Princeton University Press, 2008.

4 Voir Douglass North, Institutions, Institutional Change and Economic Performance, Cambridge, Cambridge University Press, 1990; James Mahoney et Katheleen Thelen (dir.), Explaining Institutional Change. Ambiguity, Agency and Power, Cambridge, Cambridge University Press, 2010.

5 "Historicism (or historism in this translation of Curtius' Historismus) is a critical movement insisting on the prime importance of historical context to the interpretation of texts of all kinds". Nous avons ici plutôt à l'esprit l'idée suivante: "Historicism emerges in reaction to the practice of deducing from first principles truths about how people are obliged to organize themselves socially and 
connaissance historique (le déroulement des évènements tels que transcrit par le travail des historiens) qui nous fait prendre conscience que la modélisation dominante en économie donne trop de poids à l'équilibre, la continuité, la stabilité. Comme l'a rappelé la récente crise financière, le choc, la rupture, la brusque discontinuité peuvent survenir en économie ${ }^{6}$. Une modélisation qui tend à donner trop peu de poids aux évènements extrêmes peut mener l'économiste à gravement sous-estimer les risques systémiques et l'instabilité du système qu'il étudie.

Nous nous proposons donc, dans une première partie, de rappeler les grandes critiques d'une science économique dominante fondée sur une analogie physique (voire mathématique), avant de passer à la définition des notions d'histoire et d'historicité. Nous passerons alors en revue les tentatives faites pour réintroduire ces concepts au cœur de la modélisation économique elle-même en mettant l'accent sur les apports d'une cliométrie théorique. On parlera de la théorisation des institutions par North et de la modélisation de la notion de dépendance à la trajectoire par David. Nous montrerons aussi comment certains économistes ont essayé, en recourant aux outils les plus standards de la science économique, de rendre compte par une modélisation unique de la trajectoire de long terme des économies (la croissance unifiée par Galor). On notera aussi la pertinence des réflexions économétriques en rappelant les approches des séries temporelles. Un des apports essentiels de ces nouveaux travaux d'économétrie historique est justement de distinguer ces chocs qui auront un impact permanent (justifiant par là le fait que l'histoire compte) ou seulement transitoire (là l'histoire devient moins essentielle). Nous conclurons en plaidant pour rendre la modélisation mathématique et les outils économétriques aptes à rendre compte de l'idée que l'histoire (parfois) compte. Nous tendons à suggérer que d'une part certains économistes reprennent l'histoire au sérieux (mais plus comme une réserve de faits

politically. (Paul Hamilton, Historicism: The New Critical Idiom, New York, Routledge, 1996, p. 2).

6 Voir Carmen Reinhart et Kenneth Rogoff, This Time is Different: Eight Centuries of Financial Folly, Princeton, Princeton University Press, 2009. 
stylisés dont la théorie devrait pouvoir rendre compte dans leur succession historique) et que d'autre part des visions-clés issues des études historiques (idée que l'histoire pèse sur le présent, mais aussi qu' elle réserve des surprises sous formes de discontinuités) sont reprises et modélisées dans le langage propre (mathématisé) des économistes. Bien sûr, au final, quand nous discuterons des possibilités de prévision, nous devrons dire que l'on ne peut totalement s'abstraire de la connaissance de faits extérieurs aux modèles, et que l'on ne peut ignorer ce qui n'est pas modélisable (hélas, dirait un puriste). C'est en ce sens que nous ne pourrons complètement évacuer la notion d'historicité au sens philosophique du terme ${ }^{7}$. Pour agir ou prévoir, l'économiste doit en effet dépasser la connaissance contenue dans les modèles et être capable d'intégrer de façon incertaine d'autres dimensions. Comme Hayek, nous ne croyons pas en la capacité pour les économistes de parvenir à intégralement modéliser la réalité économique au point de pouvoir par les seuls outils mathématiques piloter et prévoir le comportement des économies.

\section{L'ambition d'une théorie économique comme physique ou mathématique sociale}

La tendance dominante en science économique (écoles classique jusque 1870 et néo-classiques depuis lors) a cherché à recourir aux méthodes et aux outils des sciences physico-mathématiques (surtout celles de la physique classique), et à partir à la recherche de relations d'équilibre stables. Elle a aussi importé la vision natura non facit saltum (la nature ne fait pas de saut), privilégiant la continuité, les tendances lourdes, à l'analyse de brusques discontinuités ${ }^{8}$. Parlant de l'homme et de la société, l'économie en contient nécessairement une représentation, mais très schématique (bien plus d'ailleurs dans l'école néo-classique que dans l'école classique qui, elle, parlait encore de classes sociales et

$7 \quad$ Il faut aussi noter chez les économistes le retour chez certains d'un désir de retour à l'histoire sans faire retour sur la modélisation (McCloskey, Reinhart et Rogoff...).

8 Cette vision économiste a d'ailleurs eu un écho en histoire au travers de l'École des Annales. 
modélisait une structure sociale réellement existante). Elle a voulu partir d'hypothèses les plus simples possibles en se fondant sur un postulat de comportement individuel de maximisation (rationalité instrumentale) dans un cadre institutionnel minimaliste (marchés libres, existence d'un commissaire-priseur...). L'analyse de la concurrence pure et parfaite et la recherche des conditions d'existence, d'unicité et de stabilité d'un équilibre général de tous les marchés en même temps fut longtemps son programme de travail essentiel (et toujours la référence, le first best vers lequel toute politique doit chercher à tendre). Les approches néo-classiques ont fait des micro-fondations une condition sine qua non de scientificité jusqu'en macroéconomie (rompant ainsi avec la volonté d'établir un raisonnement économique sur des agrégats - le programme de Fisher et Keynes). Plus récemment, les approches en termes de théorie des jeux ${ }^{9}$ ont cherché à conceptualiser l'économie en termes d'interaction entre acteurs rationnels (instrumentalement). Elles ont permis de mieux analyser des déviations par rapport à la concurrence parfaite et ont grandement dynamisé l'économie de la concurrence imparfaite. Elles ont fait prendre conscience de la très grande importance de la structure des jeux (et leur caractère ou non répété) - et par là sans doute du cadre institutionnel (qui peut être manipulé - devenant en ce sens une variable économique) et de l'importance de la temporalité des évènements. Malgré ces renouvellements de l'économie néo-classique, il est clair que, pour un anthropologue, un historien et la plupart des sociologues, cette approche apparaîtra singulièrement désincarnée (Durkheim va jusqu'à parler de métaphysique), et c'est sur ce point d'ailleurs que de nombreuses hétérodoxies sont nées depuis le XIX siècle : l'École Historique allemande en réaction à l'abstraction ricardienne ${ }^{10}$, la sociologie économique et histoire économique en réaction à la victoire du paradigme néo-classique en économie après le Methodenstreit. Ces approches ont voulu garder

9 Pour une introduction simple, voir Bruno Guerrien, La théorie des jeux, $3^{\mathrm{e}}$ éd., Paris, Economica, 2002.

10 Voir Geoffrey Hodgson, How Economics Forgot History: The Problem of Historical Specificity in Social Sciences, London, Routledge, 2001. 
ce que les néo-classiques, focalisés sur le seul mode de coordination et d'allocation marchand en modélisant un système stable et sans épaisseur historique ou institutionnel, semblaient exclure : l'histoire ${ }^{11}$, les rapports sociaux, le sens des actions économiques (pour les acteurs économiques), les autres formes de rationalité (Weber, Pareto) et les autres modes de gestion de la rareté que le marché. On notera que la rupture entre les écoles n'empêche pas certains économistes néo-classiques centraux de passer de l'une à l'autre approche (Pareto de l'économie mathématique à la sociologie), voire appeler à une co-existence pacifique entre économie pure, économie appliquée et économie sociale (Walras).

C'est la prétention à la fin du XIX $^{\mathrm{e}}$ siècle des économistes néo-classiques de faire de l'économie une science universelle et objective qui les a conduit à emprunter les mêmes concepts que ceux employés par la physique de leur temps et à procéder par raisonnements analogiques ${ }^{12}$. En référence à la physique newtonienne, ils ont proposé une vision mécaniste du monde dans laquelle les processus économiques semblent s'accomplir dans un monde extérieur aux phénomènes sociaux (disembededness, pour parler comme les sociologues économistes). D'où une conception universelle de l'économie de laquelle est exclu tout principe d'espace et de temps (en tous cas autre que laplacien). L'économie néo-classique est construite originellement dans l'a-temporel. Il n'est d'ailleurs pas sûr que ce soit vu par elle comme un problème, car son questionnement premier porte sur les conditions de fonctionnement d'une économie de marché dans un cadre idéal (une forme de mécanique rationnelle du social). L'absence d'analyse des institutions autres que marchandes ou de la dépendance au temps n'est pas pour elle un problème - ce n'est tout simplement pas son objet de recherche. Au départ, même

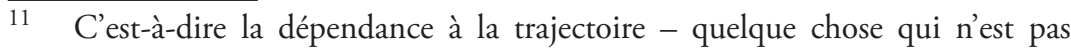
compatible avec le maintien permanent d'un équilibre stable.

12 De nombreux concepts seront d'ailleurs empruntés à la mécanique, telles que les notions de stabilité, d'élasticité, d'expansion, de frottement, etc. Voir Philip Mirowski, More Heat than Light. Economics as Social Physics, Physics as Nature's Economics, Cambridge, Cambridge University Press, 1989, p. 224. 
la croissance économique n'est plus étudiée par l'économie néoclassique (qui s'écarte en cela fort de l'économie classique), et il faudra attendre le modèle de Von Neumann, en 1938, et surtout les recherches qui suivent la Seconde Guerre mondiale pour voir cet objet faire retour, non sans problèmes. L'écho de l'école classique avec David Ricardo, en 1817, reste cependant bien là par exemple dans le modèle de Solow en 1956 où ce sont in fine les comportements optimisateurs, la compatibilité avec l'équilibre général et les hypothèses sur la technologie (fonction de production à rendements décroissants) qui sont centrales. Le rôle des institutions est au départ exclu (elles ne sont pas vues comme des faits économiques). Il faudra attendre North en 1990 pour voir revenir en force l'interrogation sur le rôle de celles-ci. Toute la science économique n'est cependant pas unanime (que ce soient les modèles de croissance endogène ou les approches historiquement plus pertinentes de Galor ${ }^{13}$, beaucoup continuent à voir les institutions comme secondaires ou au moins endogènes, causées par le développement économique).

On aura compris aussi que l'économiste hésite entre le rôle normatif ou positif. Dit-il ce qui est ou ce qui doit être? Ou s'interroge-t-il seulement sur ce qui est dans un univers virtuel, comme le mathématicien? Bref, il n'est pas sûr que l'économiste se veuille toujours le physicien du social, mais peut-être aussi un mathématicien de sociétés idéales, voire un philosophe politique ${ }^{14}$. Comme déjà souligné plus haut, les économistes ne peuvent se passer d'une certaine représentation du système social, même extrêmement pauvre et schématique, dont l'homo aconomicus rationnel constitue le centre et où tout se fonde sur des marchés et des contrats. Un physicien y verrait l'écho d'une vision atomiste. On retrouverait ici la marque d'une vision que d'aucuns nommeront philosophique (voire libérale) et d'autres axiomatique ${ }^{15}$.

13 Oded Galor, "From Stagnation to Growth: Unified Growth Theory ", dans Philippe Aghion et Steven Durlauf (dir.), Handbook of Economic Growth, Amsterdam, North-Holland, 2005, p. 171-293.

14 Voir Serge Christophe Kolm, Philosophie de l'économie, Paris, Seuil, 1986.

15 Voir Bernt Stigum, Toward a Formal Science of Economics, Cambridge, MIT Press, 1990. 
Dans cette dernière perspective, l'économiste se rapprocherait plus du mathématicien (souci de la cohérence logique et de construire un nouveau corps de savoir de façon purement déductive) que du physicien (même si dans cette discipline une telle ambition existe et si l'économie néo-classique s'est voulu le pendant social de la mécanique rationnelle). La science économique dominante chercherait ainsi à explorer toutes les conséquences logiques possibles d'un axiome de base : l'homme, dans la sphère économique ${ }^{16}$ chercherait à maximiser ses intérêts sous des contraintes de réalité. La société ne serait que le résultat de l'interaction entre individus rationnels (rationalité instrumentale). Cet axiome, qui semble faire sens - être la marque du bon sens - dans la sphère économique, permet la mobilisation des outils mathématiques de la théorie de l'optimisation. Mais il rend aussi l'économie proche d'une certaine vision libérale (hypothèse de la rationalité individuelle, ordre social naissant du chaos apparent des intérêts particuliers, auto-organisation...). L'autonomie (et même la Wertfreiheit) de l'économie n'est donc qu'apparente.

\section{Les doutes de la science économique comme mécanique rationnelle du social}

Les économistes croient que le degré d'avancement, de maturité, reconnu - mais aussi contesté - à la discipline économique est aujourd'hui lié à sa capacité de modéliser, c'est-à-dire d'utiliser les mathématiques dans la formulation et la résolution des problèmes qu'elle se pose (et qui sont par ailleurs - ou devraient être-d'une importance vitale pour les sociétés et les gouvernements). On notera tout de suite que pour certains critiques, ce ne sont pas tant les mathématiques qui feraient le succès de l'économiste

$\overline{16}$ Pour Adam Smith, 1759, 1776; mais dans toutes les sphères pour Gary Becker, par exemple. Voir Gary Becker, Human Capital. A Theoretical and Empirical Analysis with Special Reference to Education, New York, National Bureau of Economic Research, 1964; Adam Smith, The Theory of Moral Sentiments, Glasgow Edition of the Works and Correspondence of Adam Smith, Oxford, Clarendon Press, 1976 [1759]; Adam Smith, An Inquiry into the Nature and Causes of the Wealth of Nations, Glasgow Edition of the Works and Correspondence of Adam Smith, Oxford, Clarendon Press, 1976 [1776]. 
que la théorie néo-classique sous-jacente elle-même et son a priori favorable au marché - qui serait en phase avec l'esprit du temps et le souci de fonder scientifiquement les politiques menées. Ceci dit, il est vrai que l'ambition asymptotique de tout économiste est de rendre compte d'une gamme de phénomènes économiques et de plus en plus sociaux de plus en plus larges par la théorie néo-classique. L'économie ne se définit-elle pas de plus en plus comme une théorie mathématique des phénomènes socioéconomiques? Ce faisant on pourrait la qualifier de " physique sociale ", expression du belge Quételet à laquelle Comte substituera celle de sociologie ${ }^{17}$, parce qu'à la différence du premier il ne croit pas à l'application des probabilités dans le champ des phénomènes sociaux. À ce titre, l'économie peut apparaître pour certains esprits comme la plus mûre, la plus avancée, des sciences humaines et sociales (c'était un peu l'idée de Samuelson quand, dès l'introduction de son célèbre manuel Economics, il parlait de " reine des sciences sociales ») - même si certains déplorent cette assurance $^{18}$.

Mais, paradoxalement peut être, au-delà d'une très forte assurance et d'une institutionnalisation solide, les recherches en économie traversent une crise de renouvellement (déjà soulignée par Edmond Malinvaud ${ }^{19}$ ). Le fond social sur lequel nous vivons s'ébranle et se modifie profondément, à l'image peut-être de la crise précédente, en 1929, qui a vu le développement de la macroéconomie keynésienne, nouvelle forme d'économie politique en continuité avec l'esprit des mercantilistes. Sous l'action des réalités quotidiennes, les vérités qui semblaient les mieux assises ont été remises en question. L'économie est sommée de répondre aux grandes questions de notre temps (crise économique et financière, chômage croissant, globalisation, mise en question de nos modèles sociaux, question du développement...) et parfois

\footnotetext{
$17 \quad$ Dans la $47^{\mathrm{e}}$ leçon des Cours de philosophie positive, Paris, Hermann, tome 2, 1975 [1830-1842].

18 Voir Antoine (d') Autume et Jacques Cartelier (dir.), L'économie devient-elle une science dure?, Paris, Economica, 1995.

19 Voir Malinvaud, Edmond, "Pourquoi les économistes ne font pas de découvertes ", Revue d'Économie Politique, vol. 106, 1996, p. 929-942.
} 
elle peine à offrir des réponses, elle qui a tendance à raisonner dans un monde virtuel dont le temps historique, le changement irréversible est exclu. Les réponses qu'elle donne sont issues de modèles trop simplifiés, et qui peuvent ne pas être pertinents, voire dangereux, une fois appliqués dans le monde réel. Le récent débat sur l'opportunité de la rigueur budgétaire en période de croissance molle est, en la matière, une criante illustration.

C'est que la mathématisation implique des contraintes. L'économiste voit le social et l'économique comme des mécaniques d'interactions individuelles dans un cadre institutionnel figé (sauf dans cette littérature qui s'intéresse à l'émergence endogène des institutions) et en tous cas extrêmement schématiques (par la force des choses : son sujet de généralité lui enjoint de ne pas prêter attention à la contextualité et aux particularismes institutionnels locaux). La science économique néo-classique est forte localement pour analyser les (ou certains) marchés dans des situations stables. Elle l'est beaucoup moins dans les périodes d'instabilité - là où on fait le plus appel à elle paradoxalement. L'obsession de la science économique (écho de son modèle : la physique classique) pour l'équilibre, la stabilité et un certain modèle de comportement (rationalité instrumentale, comportement d'optimisation sous contraintes, anticipations rationnelles) peuvent la handicaper lourdement à moins de prendre au sérieux le message envoyé par d'autres sciences sociales (souvent des parties de la science économique qui ont pris leur autonomie suite à la victoire de l'approche néo-classique : sociologie économique, histoire économique...) : il faut tenir compte du temps historique, de la complexité, de l'incertitude radicale - toutes caractéristiques qui ne sont pas aisément modélisables. Mais c'est pourtant l'existence de ces caractéristiques centrales des phénomènes sociaux qui expliquent le rôle central joué par les institutions. Tout un courant de pensée en science économique a cherché à renouveler la discipline en y réintroduisant le rôle des institutions. Ce n'est pas un hasard si en 1993 Fogel et North puis Williamson (en 2009) ont obtenu le prix Nobel d'économie. On notera cependant que la plus grande richesse conceptuelle de 
ces approches se caractérise aussi par une moindre mathématisation et un rapprochement avec certains courants des sciences sociales et politiques ${ }^{20}$.

\section{Une nécessité : prendre l'histoire et l'historicité au sérieux en sachant de quoi l'on parle}

La validité des énoncés théoriques dépend à la fois de la cohérence externe de ceux-ci, c'est-à-dire de leur conformité avec les faits réels et de leur cohérence interne (leur cohérence logique et leur inscription dans l'ensemble de la théorie développée jusque là). La récente théorie de la croissance endogène est, à cet égard, très illustrative. Elle a suscité de nombreux travaux (théoriques) quant à la validité de ses prémisses et de ses enseignements. Mais, en revanche, les recherches consacrées à tester sa validité empirique n'ont pas totalement réussi à clore le débat sur la pertinence de ses prescriptions de politique économique (pensons aux politiques éducatives optimales par exemple, même si le paradigme néo-schumpétérien semble s'imposer auprès des décideurs $\left.^{21}\right)$. Dans cette optique, on peut toujours discuter de la cohérence d'une proposition théorique, mais il est plus important encore de la soumettre au test de la vérification empirique. Ainsi, l'excellence d'un développement théorique doit être jugée aussi en vertu de sa soumission à l'expérimentation (en économie au test économétrique). Elle ne résulte guère d'une harmonie occasionnelle entre le sensible et l'intelligible : elle doit se juger en fonction de sa capacité à résister aux tests qui ont cherché à la falsifier sur la longue durée. Dans une telle perspective, l'économie appliquée manque, à l'évidence, d'exigence.

Prenons un exemple, celui du rôle de l'éducation dans le processus de la croissance économique. Force est de constater qu'avec le XXIe siècle l'on ne sait toujours pas quelle est la nature

\footnotetext{
20 Voir James Mahoney et Katheleen Thelen (dir.), Explaining Institutional Change..., op. cit.

21 Voir Philippe Aghion et Peter Howitt « Growth with Quality - Improving Innovations: An Integrated Framework», dans Philippe Aghion et Steven Durlauf (dir.), Handbook of Economic Growth, Amsterdam, North-Holland, 2005, p. 67-110.
} 
de cette relation. Les hypothèses sont nombreuses et les estimations numériques abondantes ${ }^{22}$. On observe, d'un côté, des procédures d'analyses assez formalisées, mais dans l'ensemble peu satisfaisantes et, d'un autre côté, un corps de remarques ou d'intuitions plus justes, mais insuffisamment précises pour qu'elles puissent fonder des décisions permettant de guider une stratégie de croissance. Galor ${ }^{23}$ a montré aussi que toutes les théories de la croissance (tant classiques que néo-classiques) ne se focalisaient que sur un certain nombre de faits stylisés propres à la période de croissance soutenue du PNB par tête connue au cours du $\mathrm{XX}^{e}$ siècle. Un regard plus historique permet de se rendre compte que l'histoire économique de très long terme se caractérise par des phénomènes plus riches (très longue période de stagnation malthusienne; croissance très lente mais de plus en plus rapide néanmoins suite au progrès technique et des connaissances, elles-mêmes corrélées avec l'état d'avancement des connaissances et la densité de la population accroissant les interactions et donc les probabilités de découvertes; progrès technique qui se traduit à son tour par une demande de travail de plus en plus qualifiée jusqu'à un seuil où les familles économiquement rationnelles finissent par sortir du piège malthusien et préfèrent la qualité des enfants à leur quantité - cette croissance du capital humain favorisant à son tour l'innovation) qu'il cherche à modéliser au travers d'une théorie unique (la théorie de la croissance unifiée) qui rendrait compte de la mécanique de long terme de l'histoire économique de l'humanité. La prise de conscience historique pousse ainsi l'économiste, tout en restant fidèle à sa "boîte à outils ", à modéliser des faits stylisés plus réalistes, donnant ainsi plus de solidité empirique à ses théories.

Pour l'avenir, l'effort principal de la science économique

22 Voir Alan Krueger et Mikael Lindahl, "Education for Growth: Why and Whom? ", Journal of Economic Literature, vol. 39, 2001, p. 1101-1136; Lant Pritchett, "Where Has All the Education Gone? ", World Bank Economic Review, vol. 15, 2001, p. 367-391; Jérôme Vandenbussche, Philippe Aghion et Costas Meghir, "Growth, Distance to Frontier and Composition of Human Capital ", Journal of Economic Growth, vol. 11, 2006, p. 97-127.

23 Oded Galor, "From Stagnation to Growth: Unified Growth Theory ", op. cit. 
devrait donc être non d'y réintégrer les dieux (André Malraux) mais d'y réintégrer la dimension d'historicité. Ce dernier terme mérite une explicitation. Il faut bien avoir à l'esprit que nous n'entendons pas par là exactement la même chose que nos collègues philosophes ou des études littéraires (literary studies ${ }^{24}$ ).

On ne cherche pas ici à recontextualiser la théorie économique vue comme un ensemble de textes, ni simplement à compléter les travaux des économistes très mécanistes et a-temporels par une connaissance historique qui serait vue comme un savoir en soi, complémentaire mais autre que scientifique (en ce sens que l'histoire traditionnelle ne cherche pas à dériver des lois du fonctionnement des sociétés humaines), un peu comme le roman ou la poésie dans leur registre (source d'une forme de connaissance sur l'homme mais pas celle de la science telle qu'elle se définit selon la vision très moderne qu'en ont les économistes). Certes, c'est bien sûr là aussi un apport essentiel de l'histoire : confronter l'économiste au réel, lui faire prendre conscience de la dynamique des phénomènes et l'intégration des dimensions sociales, politiques, culturelles et économiques (car si l'histoire est moins analytique que l'économie elle est une discipline de synthèse). On a noté plus haut comment chez Galor la connaissance même stylisée (et sans doute contestable dans les détails) de l'histoire économique de longue durée l'a amené à contester la validité universelle de modélisations de la croissance adaptées à une très courte période de l'histoire humaine. L'histoire a ici constitué un aiguillon pour développer une meilleure théorie (même si cette dernière ne prend pas réellement au sérieux les remarques des approches institutionnalistes : Galor utilise de façon ingénieuse les outils néo-classiques existants et restent dans la philosophie sous-jacente du primat de la technologie. Pour lui les institutions sont endogènes et suivent le développement de l'économie).

Aller un pas plus loin consiste à prendre réellement au sérieux dans la théorie et la modélisation économique l'histoire comme dépendance à la trajectoire (et l'idée que la connaissance de la

24 Voir Paul Hamilton, Historicism..., op. cit. 
trajectoire passée est centrale pour comprendre le présent et les futurs probables). La fin des années 1980 et le début des années 1990 ont été très riches de ce point de vue avec les théories de North sur le changement institutionnel et la performance économique, les apports de David sur la dépendance à la trajectoire et les réflexions très utiles des économètres pour la compréhension de la causalité en histoire (voir pour les rapports entre histoire et économétrie la synthèse de Grenier).

\subsection{Théorie néo-institutionnaliste de North (1990)}

Douglas North considère comme central le rôle des institutions. Il donne à celles-ci une définition très abstraite (inspirée de la théorie des jeux) de règles du jeu gouvernant l'interaction sociale et rendant l'échange possible en réduisant les coûts de transaction. Il inclut dans ces institutions l'ensemble des normes formelles (par exemple le droit, les langues...) mais aussi informelles (normes sociales, culture...). Il a une approche assez fonctionnaliste de leur naissance en ce sens que pour lui les sociétés ont besoin d'institutions qui homogénéisent normes et comportements afin de réduire les coûts de transaction. Tout groupe humain générera donc des institutions à un moment donné du temps. North parle également d'organisations qui sont des groupes humains (entreprises, partis politiques, écoles...) qui vont prendre appui sur ces institutions pour faire avancer leurs intérêts - stabilisant celles-ci. En clair, les institutions font l'objet d'un processus d'auto-renforcement (elles sont donc assez stables et difficiles à changer). Pour North, le mérite des institutions est d'exister pour rendre les échanges possibles. Cependant elles incluent aussi des incitations - et c'est l'histoire qui va le mettre en évidence. Certaines sociétés ont choisi à un moment donné du temps des institutions créant davantage d'incitations pour des activités contemplatives ou d'extraction de rentes tandis que d'autres mettent en place davantage d'incitations à la création de richesses. Ceci exerce par exemple un impact direct sur les investissements en capital humain. Une fois les institutions stabilisées, il y a un effet de dépendance à la trajectoire : certaines sociétés 
ou cultures auront un avantage économique sur d'autres (les sociétés anglo-saxonnes ou protestantes - on sent ici une forme de vision moderniste téléologique qui fera bondir l'historien contemporain) du fait de ces incitations implicites mises en place à l'origine (souvent sans conscience des effets dynamiques). Cette vision de l'économie donne aux institutions tout à la fois une origine endogène à l'économie (elles naissent car elles sont utiles à l'échange) mais du fait de la dépendance à la trajectoire et des incitations mises en place elles vont exercer une action causale sur le développement économique. Certaines sociétés pourraient ainsi être piégées dans une " trappe de sous-développement " du fait de " mauvaises " institutions ou cultures. On peut voir là un argument en faveur de politiques de changement institutionnel brusque et volontariste qu'on a pu observer dans l'histoire avec un succès relatif (moins en Russie d'abord sous Pierre le Grand puis sous Staline, deux leaders politiques soucieux de moderniser leur société par l'importation d'un modèle occidental ou théorique pour mettre la nation sur de meilleurs rails de développement; davantage de succès au japon avec les réforme de l'ère Meiji à partir de 1867).

3.2. La théorisation de Paul David sur la dépendance à la trajectoire et autres remises en question de l'intérieur de la vision néo-classique " optimiste »

Paul David est un historien économiste et un économiste qui a (avec d'autres, pensons à Alan Kirman et son modèle sur les "fourmis ») travaillé sur les questions des innovations ${ }^{25}$. Il se situe en partie dans la lignée plus large des "économies de réseau " (network externalities) qui au sein du paradigme néo-classique montre qu'il peut y avoir un avantage à acquérir par exemple un appareil de communication (téléphone, ordinateur...) d'un certain type si certains en possèdent déjà un. On peut montrer que c'est le cas même s'il existe sur le marché un meilleur produit. Il y aurait donc de ce point de vue un avantage à être le premier

25 Paul David, "Path Dependence: A Foundational Concept for Historical Social Science », Cliometrica, vol. 1, 2007, p. 91-114. 
sur le marché (du moins : le premier à être acquis par un consommateur). Si deux technologies (A et B) existent, et que disons la technologie $\mathrm{A}$ est inférieure à la technologie $\mathrm{B}$, si un consommateur choisit (imaginons de façon aléatoire) la technologie A, tous les autres auront intérêt à l'acquérir, malgré son infériorité technique. Cette petite histoire simple montre plusieurs choses : l'histoire compte (l'évènement " historique " du premier choix par un consommateur conditionne tout l'historique futur - et donc le présent ne peut vraiment se comprendre qu'à la lueur du passé, voire de l'analyse précise d'un évènement - ce qui redonne évidemment ses lettres de noblesse à l'analyse historique traditionnelle en économie ${ }^{26}$ ) et les produits qui triomphent sur le marché ne sont pas nécessairement les meilleurs. Bien qu'exprimées dans le langage de l'économie néo-classique, on sent bien ici qu'on aboutit à des conclusions à la marge de l'hétérodoxie (l'histoire compte, la libre concurrence n'aboutit pas nécessairement au triomphe du meilleur...).

Les décennies des années 1980 et 1990 ont vu se développer d'autres approches qui ont contesté de façon interne le bel ordonnancement qui existait jusque là (l'influence considérable de la vision de la concurrence pure et parfaite - qui trouve un écho politique dans les politiques néo-libérales des années 1980 90 par ailleurs). La notion de rendement croissant est explorée par les économistes, de même que les scénarios de concurrence imparfaite. Par ce biais, avec les outils de la théorie néo-classique, et aussi avec le recours croissant à la théorie des jeux, on voit réapparaître des arguments qui étaient en partie ceux de l'École Historique Allemande sur certaines dimensions. Krugman ${ }^{27}$ s'interroge sur la pertinence du libre-échange total. Brander et Spencer ${ }^{28}$ montrent que l'avantage comparatif peut être construit

26 Voir Avner Greif, "Cliometrics after Forty Years », American Economic Review, vol. 87, 1997, p. 400-403.

27 Paul Krugman, «Is Free Trade Passe? ", Journal of Economic Perspectives, vol. 1, 1987, p. 131-144.

28 James Brander et Barbara Spencer, "Export Subsidies and International Market Share Rivalry ", Journal of International Economics, vol. 18, 1985, p. 83-100. 
via des subsides publics. La nouvelle économie géographique permet de réfléchir aux effets des économies d'agglomération et relativise la vision optimiste des économistes néo-classiques : dans un certain nombre d'activités, on assiste plus à une concentration qu'à une dispersion sur le territoire (avec les risques induits en termes d'inégalités).

Le point commun de toutes ces approches est de partir des grandes hypothèses de la concurrence pure et parfaite et de les lever, de façon à rendre compte de façon plus pertinente de la réalité. Bien souvent, c'est la connaissance historique (ou simplement factuelle) qui pousse certains économistes à réviser leurs modèles et par ce biais à revenir à certaines grandes intuitions critiques du passé. C'était déjà vrai pour North (après tout, l'idée d'un blocage du développement dû à des institutions inappropriées - qui n'évoluent pas car certains groupes n'ont pas intérêt à cela - était déjà bien présente chez Marx qui explorait le décalage entre une évolution continue des forces productives et un blocage lié aux rapports de production inadaptés); c'est encore le cas ici où les politiques commerciales stratégiques sont étudiées et où certaines intuitions de Friedrich List se retrouvent remises en avant ${ }^{29}$.

3.3. Apports de l'économétrie des séries temporelles sur comment penser le rôle de l'histoire en économie

L'économétrie est née pendant la crise des années 30 afin de donner à l'analyse macroéconomique (elle-même une discipline née à cette époque, corollaire de la grande crise) des fondements empiriques solides. Grâce à la quantification, et à la constitution de séries chronologiques de grandes variables économiques, et grâce à l'apport de la modélisation mathématique pour exprimer de façon non ambigüe (du moins l'espérait-on) les relations causales entre variables telles qu'elles peuvent se déduire de la

29 Voir Jean-Luc Demeulemeester et Jean-Christophe Defraigne, « Le Système National de List : la fondation du réalisme plurisdisciplinaire en économie politique internationale contre le libre-échangisme anglo-saxon ", dans Alain Alcouffe et Claude Diebolt (dir.), La pensée économique allemande, Paris, Economica, 2009, p. 227-243. 
théorie, l'économétrie allait permettre de départager les visions concurrentes et faire tendre l'économie politique vers un plus haut niveau de scientificité (ses énoncés pouvant être falsifiés pour reprendre l'expression de Popper). Bien entendu, cette discipline n'avait pas que des buts scientifiques éthérés : elle devait aussi aider les gouvernements à bâtir des politiques économiques davantage fondées (le tout dans le cadre d'une philosophie relativement interventionniste). Elle a suscité bien logiquement de puissants développements dans la constitution de bases de données à l'échelle nationale et poussé au développement de la comptabilité nationale. C'est bien entendu une philosophie économique plutôt keynésienne qui se trouvait derrière cette approche qui a connu après la seconde guerre mondiale ses heures de gloire. Ce fut l'époque des grands modèles macro-économétriques et de la modélisation structurale ${ }^{30}-$ qui fut dans un sens une mobilisation de l'histoire (l'économétrie recourant à l'époque essentiellement à des séries chronologiques de variables macro-économiques sur base annuelle, trimestrielle ou mensuelle) pour tester la pertinence et la stabilité de représentations schématiques du fonctionnement des économies. Cette économétrie-là a d'ailleurs suscité tout un travail chez les historiens quantitativistes qui ont cherché à reconstituer les comptes nationaux de façon rétrospective sur le très long terme. En parallèle, la modélisation économétrique a permis aux économistes et historiens de réfléchir davantage à des notions comme le système (une relation économique faisant partie d'un ensemble - il est dès lors central de replacer celle-ci dans le cadre de la modélisation du système dans sa totalité), le changement (les économètres cherchant à tester la stabilité des relations, ou identifier des ruptures). L'approche macro-économétrique connut un grand succès tant que l'économie restait stable (années 1950 et 1960). La crise des années 1970 va engendrer un profond mouvement de contestation de la pertinence tant des approches keynésiennes que des modèles macro-économétriques qui sem-

30 Voir Adrian Darnell et J. Lynne Evans, The Limits of Econometrics, Aldershot, Edward Elgar, 1990. 
blaient trop agrégés, sans modélisation explicite des comportements microéconomiques (donc incapables de rendre compte d'une modification des comportements suite à des changements dans la politique économique - voir la célèbre critique de Lucas ${ }^{31}$ ) et sans attention suffisante accordée à la dynamique temporelle. Léconométrie des années 1980 et 1990 s'est donc davantage orientée vers une modélisation relativement a-théorique (measurement without theory selon ses critiques; mais cela dépend des courants) et beaucoup plus statistique, concentrée sur les propriétés dynamiques des séries temporelles. Ce fut l'époque de gloire de l'économétrie des séries temporelles : approches VAR ${ }^{32}$, causalité, cointégration $^{33}$, etc. À nouveau, le côté relativement indépendant de ces approches économétriques par rapport à une méthodologie néo-classique obsédée de fondements microéconomiques et d'équilibres ne pouvait que séduire les esprits plus " historicistes " de type quantitatif. Ce n'est sans doute pas un hasard si Grenier ${ }^{34}$ a au milieu des années nonante vanté les mérites de ces approches pour l'historien.

De façon plus philosophique, ces approches économétriques font aussi réfléchir l'économiste sur la place de la dimension d'historicité. Ce qui distingue sans doute l'histoire des autres sciences sociales et économiques, c'est la place donnée à l'idée de rupture brusque et d'évènement rare. La recherche économétrique a justement redonné à ces questions leurs lettres de noblesse. Diverses approches comme l'identification de ruptures structurelles dans les séries ou l'identification de points atypiques (chocs)

31 Voir Robert Lucas, "Econometric Policy Evaluation: A critique », dans Karl Brunner et Alan Meltzern (dir.), The Phillips Curve and Labor Markets, Carnegie-Rochester Conference Series on Public Policy 1, Amsterdam, North Holland, 1976, p. 19-46.

32 Voir Christopher Sims, "Macroeconomics and Reality ", Econometrica, vol. 48, 1980, p. 1-48.

33 Voir Robert Engle et Clive Granger, "Cointegration and Error-Correction: Representation, Estimation and Testing ", Econometrica, vol. 55, 1987, p. 251-276.

34 Voir Jean-Yves Grenier, "L'histoire quantitative est-elle encore nécessaire? ", dans Jean Boutier et Dominique Julia (dir.), Passés recomposés. Champs et chantiers de l'Histoire, Paris, Autrement, série Mutations, ${ }^{\text {os }} 150-151,1995$, p. 173-183. 
a permis de mieux réfléchir à l'impact de phénomènes historiques rares (krach boursier, guerres...) sur la dynamique longue des séries économiques. Ainsi, par exemple, Darné et Diebolt ${ }^{35}$ ont-ils distingué des effets de changements de niveau ou de pente, des effets transitoires ou permanents. La recherche sur les racines unitaires en statistique et économétrie va dans le même sens : soumise à un choc, une série va-t-elle être affectée de façon seulement temporaire (effet à court terme), subir une lente résilience (effet brusque puis lent retour à la normale), ou au contraire subir un changement permanent. La cliométrie, qui cherche à réconcilier histoire, économétrie et théorie économique est très certainement une voie d'avenir et de renouveau pour prendre en compte dans la méthodologie économique les intuitions issues des études historiques (ou du moins certaines d'entreelles).

\section{Rôle central de la cliométrie pour renouveler le champ de l'économie}

Comme il ne s'agit pas de détruire l'édifice néo-classique qui a été construit, mais plutôt de l'aménager (voire en sortir à la marge) en prenant consciemment en compte l'histoire (et la conception du temps irréversible), cet effort devrait être dirigé vers la démarche cliométrique. Cela signifie d'une part de tester, plus systématiquement et sur le long terme, la validité (stabilité) des énoncés théoriques (après tout, Robert Engle et Clive Granger, en 1987, ont rappelé que la théorie néo-classique produisait des relations d'équilibre de long terme) et d'autre part de bâtir des modèles théoriques (par exemple sur la croissance ou les cycles) davantage en adéquation avec les faits stylisés dégagés d'une analyse sérieuse de l'histoire économique. La théorie n'a, en effet, pas vocation première à raisonner sur des mondes imaginaires.

Cette ferme fondation de l'économie sur une base empirique

35 Voir Olivier Darné et Claude Diebolt, «Unit Roots and Infrequent Large Shocks: New International Evidence on Output ", Journal of Monetary Economics, vol. 51, 2004, p. 1449-1465. 
de long terme nous semble fondamentale, aussi pour l'économiste appliqué, ou le décideur au cour de l'action (l'économiste étant tourné vers l'aide à la prise de décision, à l'action, la définition de scenarii d'avenir possible reste donc chose centrale pour lui). En effet, nous pensons que le futur ne peut être conçu que dans un certain degré de continuité historique, c'est-à-dire par rapport à un passé qui l'a forgé et en fonction d'un présent qui l'explique tout en le déterminant en partie (bien sûr jamais totalement). La connaissance historique en économie doit cependant faire prendre garde aussi aux extrapolations trop simplistes car les brusques ruptures (les non-linéarités) sont aussi du domaine du possible. Il est tout aussi dangereux d'ignorer l'histoire que d'imaginer le futur à l'image de celle-ci. Certes, le présent (mais aussi le passé) peut conditionner l'avenir de la même manière que le passé explique - en partie - le présent (dès que nous quittons le monde de la théorie pure et que nous prenons au sérieux le temps historique, c'est-à-dire irréversible). Le thème de la dépendance à la trajectoire devient dès lors central. Ceci dit, n'oublions pas que les comportements économiques sont complexes, et que "les agents économiques ne sont pas stupides » (hypothèse des anticipations rationnelles, telle que décrite par Esther-Mirjam Sent ${ }^{36}$ ) - une des leçons les plus pertinentes de la Nouvelle Macroéconomie Classique. Si le passé conditionne le présent et parfois l'image qu'on se fait de l'avenir (anticipations, adaptatives dans ce cas), les anticipations de l'avenir, l'image qu'on s'en fait, peuvent à leur tour conditionner les décisions présentes. Ce serait ici le futur (la façon dont on l'anticipe) qui conditionnerait en partie le présent, et par son impact sur les décisions, la trajectoire économique future effective. Ainsi donc l'économie n'est pas le lieu de l'intemporalité, elle possède une historicité irréductible et très complexe, le passé pesant sur le présent comme l'image de l'avenir conditionne les choix actuels. Elle est, sans doute davantage en macroéconomie que dans la théorie microécono-

36 Esther-Mirjam Sent, « Sargent’s Symmetry Saga: Ontological versus Technical Constraints ", dans Mäki Uskali (dir.), The Economic Worldview: Studies in the Ontology of Economics, Cambridge, Cambridge University Press, 2001, p. 335-358. 
mique, une lecture (interprétation) de l'histoire économique influencée par le contexte présent et les nécessités de la prise de décision pour l'avenir. Les théories en macroéconomie cherchent donc à rationaliser des évènements historiques passés (crises, mouvements de croissance...) et à en rendre compte par une modélisation qui devrait aider le décideur dans le présent. On pourrait même dire qu'elle cherche à rendre compte de faits historiques par des modélisations a-historiques (jusqu'ici) - car sa vision de la science reste celle de la physique et la quête de régularités. Les approches d'économétrie (développées en parallèle de la recherche macroéconomique) cherchent à extraire le maximum d'information de séries quantitatives qui, par la force des choses, concernent toujours le passé (toute économétrie est donc toujours de l'histoire quantitative). Il faut insister sur cette dimension historique. Toute négligence sur ce plan serait à l'origine d'incompréhensions et souvent de décisions inopportunes (pour l'économiste comme " conseiller du prince »). Si l'on se contente, pour étudier un système économique, de mesurer sa composition présente, on néglige le fait que ses structures, comme ses caractéristiques (et les institutions qui l'encadrent), résultent d'une longue histoire qui influencera directement son avenir.

\section{Conclusion}

L'étude approfondie d'un système économique, surtout quand elle sert de point de départ à l'élaboration de prévisions, passe par le respect d'un certain nombre de précautions. La première d'entre elles est la capacité à prendre du recul. La référence au passé n'a pas seulement un intérêt historique, anecdotique. Les enseignements que l'on en tire sont très précieux pour éviter de généraliser trop rapidement des observations particulières, et cela d'autant plus, que l'économie s'inscrit dans la longue durée. La discipline économique ne peut pas être statique ou dynamique (au sens de statique comparative) en ne tenant pas compte de l'irréversibilité du temps. La méconnaissance des événements 
ayant abouti à la situation observée à un moment donné empêcherait d'en saisir toute la signification. L'observation du passé montre aussi, qu'il n'est pas possible de généraliser la situation vécue par une génération, de l'extrapoler dans le temps. L'historien économique ne dit pas que l'avenir est à l'image du passé. L'économiste (surtout celui qui fait face à la décision dans le monde réel) fait face à un système complexe, dont la théorie n'épuise pas tout. C'est pourquoi, nous estimons, que la connaissance historique (qui inclut des phénomènes dont nos théories ne rendent pas - encore? - compte) est indispensable pour compléter l'analyse logique fournie par des modèles qui ne sont souvent que l'abstraction de situations particulières en un temps et un lieu donné. Compte tenu de cette complexité, la discipline économique est donc certainement aussi un art et pas uniquement une science. Anticiper les tendances futures des économies relève sans doute autant de l'art, d'un mixte optimal de notre savoir théorique, de nos connaissances sur le système étudié et d'une part d'intuition, qui serait la résolution non encore consciente d'un problème hautement complexe. C'est l'art de reconnaître les points sur lesquels doit porter l'effort principal afin d'atteindre les objectifs visés. Dire qu'il s'agit d'un art, c'est admettre que les choix ne sauraient être dictés par la seule mise en œuvre de techniques statistiques sophistiquées. Aussi, prévoir l'avenir - ou plutôt les avenirs possibles - c'est d'abord fixer les objectifs que le développement des différents systèmes économiques devrait s'efforcer d'atteindre. Le futur dépend aussi de nos choix, y inclus politiques, avec la part d'idéologie ou de philosophie que cette dépendance implique. Ce choix, cette décision n'est pas complètement Wertfrei. Elle manifeste aussi des valeurs, des objectifs politiques, eux-mêmes influencés par un contexte plus large (groupes d'intérêt en présence, institutions ${ }^{37}$ ), et par l'histoire $^{38}$. Les contraintes majeures étant celles qui découlent

$37 \quad$ Voir Avinash Dixit, The Making of Economic Policy. A Transaction-Cost Politics Perspective, CES Munich Lectures in Economics, Cambridge, MIT Press, 1996.

38 Voir Douglass North, "Economic Performance Through Time ", American Economic Review, vol. 84, 1994, p. 359-368. 
des moyens financiers disponibles, de la démographie, des besoins de l'économie, de la demande sociale et des stratégies politiques.

\section{Bibliographie}

Aghion, Philippe et Peter Howitt "Growth with Quality - Improving Innovations: An Integrated Framework ", dans Philippe Aghion et Steven Durlauf (dir.), Handbook of Economic Growth, Amsterdam, North-Holland, 2005, p. 67-110.

Albert, Hans, « Modell-Platonismus. Der neoklassische Stil des ökonomischen Denkens in kritischer Beleuchtung ", dans Ernst Topitsch (dir.), Logik der Sozialwissenschaften, Köln, Kiepenheuer \& Witsch, 1970, p. 406-424.

Autume, Antoine (d') et Jacques Cartelier (dir.), L'économie devient-elle une science dure?, Paris, Economica, 1995.

Becker, Gary, Human Capital. A Theoretical and Empirical Analysis with Special Reference to Education, New York, National Bureau of Economic Research, 1964.

Blaug, Mark, La pensée économique, 5éd., Paris, Economica, 1998.

Brander, James et Barbara Spencer, " Export Subsidies and International Market Share Rivalry ", Journal of International Economics, vol. 18, 1985, p. 83-100.

Cours de philosophie positive, Paris, Hermann, Tome 2, 1975 [18301842].

Darné, Olivier et Claude Diebolt, "Unit Roots and Infrequent Large Shocks: New International Evidence on Output ", Journal of Monetary Economics, vol. 51, 2004, p. 1449-1465.

Darnell, Adrian et J. Lynne Evans, The Limits of Econometrics, Aldershot, Edward Elgar, 1990.

David, Paul, «Path Dependence: A Foundational Concept for Historical Social Science », Cliometrica, vol. 1, 2007, p. 91-114.

Debreu, Gérard, "The Mathematisation of Economic theory ", American Economic Review, vol. 81, 1991, p. 1-7.

Demeulemeester, Jean-Luc et Jean-Christophe Defraigne, "Le Système National de List : la fondation du réalisme plurisdisciplinaire en 
économie politique internationale contre le libre-échangisme anglosaxon ", dans Alain Alcouffe et Claude Diebolt (dir.), La pensée économique allemande, Paris, Economica, 2009, p. 227-243.

Dixit, Avinash, The Making of Economic Policy. A Transaction-Cost Politics Perspective, CES Munich Lectures in Economics, Cambridge, MIT Press, 1996.

Engle Robert et Clive Granger, "Cointegration and Error-Correction: Representation, Estimation and Testing ", Econometrica, vol. 55, 1987, p. 251-276.

Friedman, Milton, Essays in Positive Economics, Chicago, University of Chicago Press, 1953.

Galor, Oded, "From Stagnation to Growth: Unified Growth Theory ", dans Philippe Aghion et Steven Durlauf (dir.), Handbook of Economic Growth, Amsterdam, North-Holland, 2005, p. 171-293.

Greif, Avner, "Cliometrics after Forty Years ", American Economic Review, vol. 87, 1997, p. 400-403.

Grenier, Jean-Yves, "L'histoire quantitative est-elle encore nécessaire?" ", dans Jean Boutier et Dominique Julia (dir.), Passés recomposés. Champs et chantiers de l'Histoire, Paris, Autrement, série Mutations, $\mathrm{n}^{\text {os }} 150$ 151, 1995, p. 173-183.

Guerrien, Bruno, La théorie des jeux, $3^{\mathrm{e}}$ éd., Paris, Economica, 2002.

Hamilton, Paul, Historicism: The New Critical Idiom, New York, Routledge, 1996.

Heijdra, Ben, Foundations of Modern Macroeconomics, $2^{\mathrm{e}}$ éd., Oxford, Oxford University Press, 2009.

Hodgson, Geoffrey, How Economics Forgot History: The Problem of Historical Specificity in Social Sciences, London, Routledge, 2001.

Hoppe, Hans, Economic Science and the Austrian Method, Ludwig von Mises Institute, 1995.

Kuhn, Thomas, The Structure of Scientific Revolutions, 2 e éd., Chicago, University of Chicago Press, 1970.

Kolm, Serge Christophe, Philosophie de l'économie, Paris, Seuil, 1986.

Krueger, Alan et Mikael Lindahl, "Education for Growth: Why and Whom? ", Journal of Economic Literature, vol. 39, 2001, p. 11011136.

Krugman, Paul, "Is Free Trade Passe? ", Journal of Economic Perspectives, vol. 1, 1987, p. 131-144.

Lakatos, Imre et Alan Musgrave (dir.), Criticism and the Growth of Knowledge, Cambridge, Cambridge University Press, 1970.

Leontieff, Wassily, "Hypothèses théoriques et faits non observés ", dans 
Bernard Rosier (dir.), Wassily Leontieff. Textes et itinéraire, Paris, La Découverte, 1986, p. 11-21.

Lucas, Robert, "Econometric Policy Evaluation: A critique ", dans Karl Brunner et Alan Meltzern (dir.), The Phillips Curve and Labor Markets, Carnegie-Rochester Conference Series on Public Policy 1, Amsterdam, North Holland, 1976, p. 19-46.

Mahoney, James et Katheleen Thelen (dir.), Explaining Institutional Change. Ambiguity, Agency and Power, Cambridge, Cambridge University Press, 2010.

Malinvaud, Edmond, « Pourquoi les économistes ne font pas de découvertes ", Revue d'Économie Politique, vol. 106, 1996, p. 929-942.

Mankiw N. Gregory, "The Macroeconomist as Scientist and Engineer ", Journal of Economic Perspectives, 20, 2006, p. 29-46.

Marx, Karl, Das Kapital, Berlin, Dietz-Verlag, Berlin, 1964 [1867].

Mingat, Alain, Pierre Salmon et Alain Wolfelsperger, Méthodologie économique, Paris, Presses Universitaires de France, 1985.

Mirowski, Philip, More Heat than Light. Economics as Social Physics, Physics as Nature's Economics, Cambridge, Cambridge University Press, 1989.

Mises, Ludwig von, Epistemological Problems of Economics, Ludwig von Mises Institute, 2003 [1933].

Morishima, Michio, Marx's Economics: A Dual Theory of Value and Growth, Cambridge, Cambridge University Press, 1973.

Neumann, John von, «Über ein ökonomisches Gleichungssystem und eine Verallgemeinerung des Brouwerschen Fixpunktsatzes ", Ergebnisse eines Mathematischen Kolloquiums, no 8, 1937, p. 73-83.

North, Douglass, Institutions, Institutional Change and Economic Performance, Cambridge, Cambridge University Press, 1990.

North, Douglass, "Economic Performance Through Time ", American Economic Review, vol. 84, 1994, p. 359-368.

Popper, Karl, La logique de la découverte scientifique, Paris, Payot, 1973 [édition allemande : 1935].

Pritchett, Lant, "Where has All the Education Gone?", World Bank Economic Review, vol. 15, 2001, p. 367-391.

Quesnay, François, "Tableau économique ", dans INED, François Quesnay et la Physiocratie, recueil, Paris, 1958 [1758].

Quételet, Adolphe, Sur l'homme et le développement de ses facultés, ou essai de physique sociale, Bruxelles, Louis Hauman, 1836.

Reinhart, Carmen et Kenneth Rogoff, This Time is Different: Eight Centuries of Financial Folly, Princeton, Princeton University Press, 2009. 
Ricardo, David, On the Principles of Political Economy and Taxation, London, J. Murray, 1817.

Roemer, John, "Exploitation, Alternatives and Socialism ", Economic Journal, vol. 92, 1982, p. 87-107.

Rosanvallon, Pierre, Le capitalisme utopique. Histoire de l'idée de marché, Paris, Seuil, 1999.

Rosenberg, Alexander, Economics: Mathematical Politics, or the Science of Diminishing Return?, Chicago, University of Chicago Press, 1992.

Rosenstein-Rodan, Paul, "The Role of Time in Economic Theory ", Economica, New Series, vol. 1, 1934, p. 77-97.

Russell, Bertrand, "Recent Work on the Principles of Mathematics ", International Monthly, vol. 4, 1901, p. 366-379.

Samuelson, Paul, Economics: An Introductory Analysis, New York, McGrawHill, 1948.

Schmoller, Gustav, Über einige Grundfragen der Sozialpolitik und der Volkswirtschaftslehre, Leipzig, Duncker \& Humblot, 1904.

Schumpeter, Joseph, History of Economic Analysis, Oxford, Oxford University Press, 1954.

Sent, Esther-Mirjam, "Sargent's Symmetry Saga: Ontological versus Technical Constraints ", dans Mäki Uskali (dir.), The Economic Worldview: Studies in the Ontology of Economics, Cambridge, Cambridge University Press, 2001, p. 335-358.

Simiand, François, Le salaire, l'évolution sociale et la monnaie. Essai de théorie expérimentale du salaire, Paris, Félix Alcan, 1932.

Sims, Christopher, "Macroeconomics and Reality ", Econometrica, vol. 48, 1980, p. $1-48$.

Smith, Adam, The Theory of Moral Sentiments, Glasgow Edition of the Works and Correspondence of Adam Smith, Oxford, Clarendon Press, 1976 [1759].

Smith, Adam, An Inquiry into the Nature and Causes of the Wealth of Nations, Glasgow Edition of the Works and Correspondence of Adam Smith, Oxford, Clarendon Press, 1976 [1776].

Snooks, Graeme Donald, Economics without Time. A Science Blind to the Forces of Historical Change, Basingstoke, Macmillan, 1993.

Snooks, Graeme Donald (dir.), Historical Analysis in Economics, London, Routledge, 1993.

Stigum, Bernt, Toward a Formal Science of Economics, Cambridge, MIT Press, 1990.

Vandenbussche, Jérôme, Philippe Aghion et Costas Meghir, "Growth, Distance to Frontier and Composition of Human Capital ", Journal 
of Economic Growth, vol. 11, 2006, p. 97-127.

Weintraub, E. Roy, How Economics Became a Mathematical Science, Durham, Duke University Press, 2002.

Wickens, Mickael, Macroeconomic Theory: A Dynamic General Equilibrium Approach, Princeton, Princeton University Press, 2008. 